\title{
Un élément fini de poutre fissurée
}

\section{Application à la dynamique des arbres tournants}

\author{
Saber El Arem — Habibou Maitournam \\ Laboratoire de Mécanique des Solides, CNRS (UMR 7649) \\ Ecole Polytechnique, F-91128 Palaiseau
}

\begin{abstract}
RÉSUMÉ. Dans ce travail on présente une méthode originale de construction d'un élément fini de poutre affectée de fissurations. La souplesse additionnelle due à la présence des fissures est identifiée à partir de calculs éléments finis tridimensionnels tenant compte des conditions de contact unilatéral entre les lèvres. Cette souplesse est répartie sur toute la longueur de l'élément dont on se propose de construire la matrice de rigidité. La démarche permet un gain considérable en temps de calcul par rapport à la représentation nodale de la section fissurée lors de l'intégration temporelle de systèmes différentiels en dynamique des structures.

ABSTRACT. In this paper, an original method for construction of a cracked beam finite element is presented. The additional flexibility due to the cracks is identified from three-dimensional finite element calculations taking into account the unilateral contact conditions between the cracks lips. Based on this flexibility which is distributed over the entire length of the element, a new cracked finite element stiffness matrix is deduced. Considerable gain in computing efforts is reached compared to the nodal representation of the cracked section when dealing with the numerical integration of differential equations in structural dynamics.

MOTS-CLÉS : fissure, poutre, contact unilatéral, éléments finis, rotor.

KEYWORDS: crack, beam, unilateral contact, finite element, rotor.
\end{abstract}

DOI:10.3166/REMN.16.643-663 (c) Lavoisier, Paris 


\section{Introduction}

Les arbres de machines tournantes constituent des structures difficiles à analyser. Ils sont souvent composés de plusieurs tronçons et soumis à des sollicitations d'origines diverses suivant les applications auxquelles ils sont destinés. Les rotors sont omniprésents dans les industries aéronautique, aérospatiale, automobile et en particulier dans l'industrie de l'énergie où l'enjeu économique est important. Aux USA, une grande partie des centrales de production d'électricité ont été construites au cours des années 1960 et dimensionnées pour une durée de vie de 30 ans (Dimarogonas, 1996). D'après Bently et al. (1986), dans les années 1970 et jusqu'au début des années 1980, au moins 28 cas de rupture d'arbres dus à la présence de fissures ont été enregistrés dans l'industrie de l'énergie aux USA malgré l'inspection des machines avant le démarrage et au cours de l'exploitation.

Depuis les années 1980, l'intérêt des chercheurs à caractériser les structures comportant des fissures ne fait que croître. Entre le début des années 1970 et la fin des années 1990, plus de 500 articles concernant les structures fissurées ont été publiés (Dimarogonas, 1996). Un résumé des principaux travaux analytiques, numériques et expérimentaux développés pour la modélisation et l'étude du comportement des structures fissurées a été présenté par Dimarogonas et al. (1983), Entwistle et al. (1990), Wauer (1990), Gasch (1993), Dimarogonas (1996), El Arem (2006).

L'étude des rotors de turbines fissurés a commencé dans l'industrie de l'énergie aux USA avec les travaux de Dimarogonas (Dimarogonas, 1970; Dimarogonas, 1971). En Europe, les premiers travaux sont apparus quelques années plus tard et font encore référence (Gasch (1976), Mayes et al. (1976), Henry et al. (1976)). Ces auteurs ont considéré un modèle simple de respiration de la fissure auquel on fait souvent référence par le "switching crack model" ou le "hinge crack model" : la fissure est soit totalement ouverte soit totalement fermée.

Zuo (1992), et Zuo et al. (1994) ont également utilisé un modèle bilinéaire pour décrire approximativement la vibration d'un arbre fissuré dans le but de développer une méthode de détection de fissures en ligne. Ils ont effectué l'étude d'un système à $1 \mathrm{ddl}$ puis ils ont examiné le comportement d'un système à $2 \mathrm{ddl}$. En se basant sur la notion de mode normal de Rosenberg, ils ont défini les modes non linéaires du système linéaire par morceaux. Ces derniers ont été mis en évidence numériquement, et dans certains cas simples, analytiquement.

Bachschmid et ses collaborateurs (Bachschmid et al., 2002; Bachschmid et al., 1980; Bachschmid et al., 2004) ont examiné les effets de la présence d'une fissure sur la réponse vibratoire d'un rotor ou d'un axe de pompe. Des modèles expérimentaux et numériques ont été proposés, les effets thermiques sur le mécanisme de respiration de la fissure ont été pris en compte dans (Bachschmid et al., 2004). Il a été rapporté que la distribution de température n'est pas influencée par la présence de la fissure contrairement à celles des contraintes et des déformations. 
Bachschmid et al. (1980) ont proposé la mise en œuvre d'une procédure itérative de résolution de l'équation d'équilibre de la section fissurée soumise aux efforts de flexion et de traction-compression pour calculer ses moments quadratiques caractéristiques. Dans ce cadre (Varé et al., 2000), il faut définir la famille de champs de contraintes statiquement admissibles où rechercher la solution. On peut craindre que cette démarche conduise à des temps de calcul relativement élevés. Cette méthode traduit également le souci de décrire finement la réponse de l'élément de rotor fissuré en fonction de l'orientation des efforts qui lui sont appliqués.

Une comparaison des différentes approches de modélisation des poutres fissurées a été présentée par Friswell et al. (2002). Les auteurs ont montré que pour la surveillance des structures basée sur les vibrations en basses fréquences, des modèles simples de flexibilité de la fissure et des éléments de type poutre sont adéquats. Cependant, la démarche de modélisation basée sur un système dynamique bilinéaire, qui fait encore référence, n'en reste pas moins approchée, ce qui amène à quelques réserves quant à la qualité des résultats quantitatifs issus de son exploitation.

Aujourd'hui, la plupart des travaux sur la vibration des rotors fissurés consistent à explorer plus profondément certains points particuliers tels que la phase d'accélération ou de décélération de l'arbre, le passage par la vitesse critique ou le couplage entre divers modes de vibrations afin d'exhiber des paramètres favorisant la détection des fissures dans le cadre d'une procédure de surveillance. Ces travaux dont on peut citer par exemple ceux de Darpe et al. (2004), ceux de Jun et Eun (Jun et al., 1992) et ceux de Sinou et al. (2005) et Sinou et al. (2007) où on trouve les résultats numériques et expérimentaux de leurs investigations sur certains points tels que cités ci-avant, restent fidèles aux principes théoriques formalisés dans le courant des années 1970 dans quelques articles fondateurs. Parallèlement, le progrès remarquable et continu de l'outil informatique permet la réalisation de performantes modélisations tridimensionnelles. Ainsi, il est devenu possible d'envisager une identification de la loi de comportement d'une section d'arbre fissurée qui s'affranchisse d'un certain nombre d'hypothèses et d'approximations faites jusqu'à présent sans dégrader les coûts des calculs de dynamique des arbres tournants.

L'objectif principal de ce travail est la présentation d'une nouvelle méthode de construction d'un élément fini de poutre comportant des fissures. La validation de la démarche sur un cas d'arbre fissuré en rotation autour de son axe est ensuite présentée. Les résultats obtenus pourraient être utiles dans le cadre d'une procédure de surveillance des arbres tournants visant la détection des fissures.

\section{Modélisation des arbres fissurés}

\subsection{Etat de l'art}

Le comportement vibratoire d'un arbre fissuré est non linéaire et assez complexe. Il nécessite, pour une description pertinente, une modélisation fine et précise de l'arbre 
et des fissures afin de permettre l'identification et le calcul des paramètres caractérisant leur présence.

Les modèles actuels de rotors fissurés reposent sur deux principes fondamentaux :

- la représentation de la section fissurée par une flexibilité locale,

- la prise en compte d'un mécanisme d'ouverture-fermeture de la fissure, encore appelé respiration.

Les premières tentatives d'analyse et de calcul de la flexibilité locale d'une région fissurée d'un élément de structure sont dues à Irwin (1957), Bueckner (1958), Westmann et al. (1967) en reliant la flexibilité locale aux facteurs d'intensité de contraintes. Dans le cas d'une structure élastique contenant une fissure de profondeur $a$, le théorème de Castigliano permet de calculer, en tout point de la structure, le vecteur déplacement additionnel $\boldsymbol{u}$ dû à la présence de la fissure sous l'action du vecteur chargement $\boldsymbol{P}$ par

$$
\boldsymbol{u}=\frac{\partial}{\partial \boldsymbol{P}} \int_{0}^{a} \boldsymbol{G}(a) d a
$$

ensuite les termes de la matrice de flexibilité locale $c_{i j}$ sont calculés par la formule

$$
c_{i j}=\frac{\partial^{2}}{\partial P_{i} \partial P_{j}} \int_{0}^{a} \boldsymbol{G}(a) d a, \quad 1 \leq i, j \leq 6
$$

$\boldsymbol{G}$ est le taux de restitution de l'énergie relié, dans le cadre de l'élasticité linéaire quasi statique et des petites perturbations, aux facteurs d'intensité de contraintes au même point $\left(K_{I}, K_{I I}\right.$ et $\left.K_{I I I}\right)$ par la formule d'Irwin (1957).

Depuis, les efforts se sont multipliés pour l'évaluation des coefficients d'intensité de contraintes pour différentes géométries de fissures et de structures. Il existe de nombreuses solutions analytiques ou semi-analytiques pour les solides fissurés infinis ou de forme extérieure simple compilées dans des recueils comme celui de Tada et al. (1973).

Liebowitz et al. (1967), Liebowitz et al. (1968) et Okamura et al. (1969) ont examiné les effets de la présence d'une rainure sur la stabilité au flambage élastique des colonnes. En combinant les résultats de la mécanique de la rupture aux résultats expérimentaux de Brown et al. (1966), ils ont proposé une formule pour l'identification de la flexibilité locale d'une poutre de section rectangulaire fissurée en flexion.

Dans deux notes techniques à la NASA, Gross et Srawley (Gross et al., 1964; Gross et al., 1965) ont identifié les coefficients d'intensité de contraintes et les termes de la matrice de flexibilité locale pour des éléments fissurés soumis à des efforts de flexion et de traction. Ils ont, en particulier, identifié les termes de couplage. De même, Rice et al. (1972) ont examiné ce couplage dans leur étude des plaques élastiques fissurées.

Dimarogonas et ses collaborateurs (Dimarogonas, 1982; Dimarogonas et al., 1983; Dimarogonas, 1987; Dimarogonas, 1988), et Anifantis et al. (1983) ont introduit la 
matrice de flexibilité $(6 \times 6)$ d'une section fissurée. Ils ont noté qu'elle contient des termes extra-diagonaux conduisant au couplage des vibrations longitudinale et transversale. En négligeant les effets de torsion, ils ont évalué les termes de cette matrice $(5 \times 5)$ pour une section rectangulaire contenant une fissure transversale. Papadopoulos et Dimarogonas (Papadopoulos et al., 1987a; Papadopoulos et al., 1987b; Papadopoulos et al., 1987c), et Ostachowicz et Krawczuk (Ostachowicz et al., 1992) ont évalué tous les termes de la matrice $(6 \times 6)$ d'une section de poutre de Timoshenko fissurée pour tout cas de chargement.

Cependant, dans le cas d'une géométrie cylindrique telle que celle des rotors de turbines, il n'existe pas d'expressions analytiques des facteurs d'intensité de contraintes, ce qui a conduit Dimarogonas et al. (1983) à discrétiser la surface fissurée en un ensemble d'éléments indépendants. Sur chacune de ces surfaces élémentaires, les coefficients d'intensité de contraintes peuvent être approchés par des formules analytiques, ensuite le taux de restitution d'énergie est déterminé par intégration.

Si elle offre l'avantage d'être facilement applicable dans un algorithme numérique, cette méthode n'en reste pas moins une approximation dont la précision n'est pas établie (Varé et al., 2000). Par ailleurs, des problèmes de convergence de la procédure d'intégration ont été mis en évidence lorsque la profondeur de la fissure dépasse le rayon de la section du rotor (Abraham et al., 1994; Dimarogonas, 1994). Enfin, la généralisation de cette démarche à toute géométrie de section fissurée est complexe, voire impossible dans certains cas où la fissure n'est pas connexe.

Pour identifier numériquement les coefficients de flexibilité locale dus à la présence d'une fissure, plusieurs tentatives ont été faites. Dirr et al. (1988), et Imam et al. (1989) ont utilisé une modélisation par éléments finis tridimensionnels avec un comportement élastique. Ostachowicz et al. (1990) ont utilisé des éléments triangulaires et un comportement élastoplastique du matériau. Go et al. (1994) ont rapporté que l'utilisation de la méthode des éléments finis pour modéliser la variation des contraintes en fond de fissure nécessite des maillages très raffinés et des temps de calculs importants tant la procédure converge lentement.

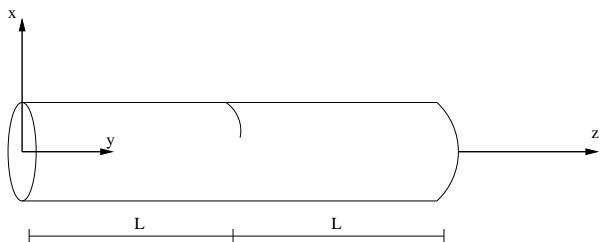

(a) Modèle tridimensionnel

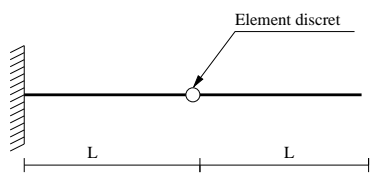

(b) Modèle poutre

Figure 1. Modélisation de l'élément de poutre fissurée

Une méthode originale de calcul de la loi de comportement en flexion d'une poutre fissurée a été proposée par Varé et al. (2000) et Andrieux et al. (2002). Elle consiste à 
identifier, à partir de calculs tridimensionnels tenant compte du contact unilatéral entre les lèvres des fissures, une relation de comportement non linéaire entre le moment de flexion appliqué à la section fissurée et le champ de déplacement résultants. Cette démarche a été validée expérimentalement par Audebert et al. (2000). Puis elle a été mise en œuvre sur plusieurs cas industriels, en particulier des turbines.

Pour l'élément fissuré de la figure 1 soumis, en $z=2 L$, au couple de moments $M_{2 L}=\left(M_{x}(2 L), M_{y}(2 L)\right)$ à l'exclusion de tout autre effort, Andrieux (2000) a démontré certaines propriétés de l'énergie élastique du problème, $W^{*}$. Elles ont permis de réduire considérablement les calculs tridimensionnels nécessaires à l'identification de la loi de comportement recherchée. En particulier, pour un matériau élastique linéaire, sous l'hypothèse des petites perturbations et en l'absence de frottement sur les lèvres des fissures : La fonction $W^{*}$ est strictement convexe et positivement homogène de degré 2. Ainsi, l'énergie peut s'écrire, en distinguant l'apport de la section fissurée et celui des éléments sains, sous la forme :

$$
W^{*}\left(\boldsymbol{M}_{2 L}\right)=W^{*}(\boldsymbol{M})=W_{s}^{*}(\boldsymbol{M})+w^{*}(\boldsymbol{M})=\frac{L}{E I}\|\boldsymbol{M}\|^{2}(1+s(\Phi))
$$

où $\boldsymbol{M}=\left(M_{x}, M_{y}\right)$ désigne le couple de moments de flexion au niveau de la section fissurée, $W_{s}^{*}(\boldsymbol{M})$ l'énergie élastique totale de l'élément sain sous le chargement $\boldsymbol{M}$ et $w^{*}(\boldsymbol{M})$ l'énergie élastique supplémentaire due à la présence de la fissure. La direction de chargement est définie par l'angle $\Phi=\operatorname{atan}\left(\frac{M_{y}}{M_{x}}\right) . s(\Phi)$ est la fonction de souplesse additionnelle due à la présence de la fissure. $E$ est le module de Young et $I$ le moment d'inertie quadratique.

Dans le cas d'une fissure à fond droit d'une profondeur $a$ telle que $\frac{a}{D} \leq 50 \%$, la fissure est totalement fermée lorsque $\Phi=\frac{\pi}{2}$. Son ouverture s'accompagne d'une évolution importante de $s(\Phi)$ conduisant à une fissure totalement ouverte lorsque $\Phi=$ $\frac{3 \pi}{2}$, cf. figure 2(a). La fonction $s(\Phi)$ ne dépend que de la direction de chargement définie par $\Phi$ : elle est indépendante de $\|\mathbf{M}\|$, cf. figure 2(b).

Comme l'illustre la figure 2(c), l'énergie apportée par la section fissurée, $w^{*}(\boldsymbol{M})$, est indépendante de $L$ pour $L \geq 2 D$ en accord avec le principe de Saint Venant (El Arem, 2006). Dans ce cadre, la relation de comportement non linéaire de l'élément discret modélisant la fissure s'obtient par dérivation de l'énergie élastique apportée par la section fissurée par rapport à $\boldsymbol{M}$.

$$
[\boldsymbol{\theta}]=\left(\begin{array}{c}
{\left[\theta_{x}\right]} \\
{\left[\theta_{y}\right]}
\end{array}\right)=\frac{2 L}{E I}\left(\begin{array}{cc}
s(\Phi) & -\frac{1}{2} s^{\prime}(\Phi) \\
\frac{1}{2} s^{\prime}(\Phi) & s(\Phi)
\end{array}\right)\left(\begin{array}{l}
M_{x} \\
M_{y}
\end{array}\right), \quad s^{\prime}(\Phi)=\frac{d s(\Phi)}{d \Phi}
$$

$[\boldsymbol{\theta}]$ désigne le vecteur des discontinuités de rotations de part et d'autre de la section fissurée. Cependant, une loi de comportement exploitable par les codes de lignes d'arbres doit être de la forme $[\boldsymbol{\theta}]=f(\boldsymbol{M})$. Ceci a conduit les auteurs à introduire une propriété de la fonction énergie de déformation élastique apportée par la section fissurée 
$w([\boldsymbol{\theta}])$ : La fonction $w$ est strictement convexe et positivement homogène de degré 2 : Cette propriété a permis d'écrire cette énergie sous la forme :

$$
w\left(\left[\theta_{x}\right],\left[\theta_{y}\right]\right)=\frac{E I}{4 L} k(\varphi)\|[\boldsymbol{\theta}]\|^{2} \text { avec } \varphi=\operatorname{atan}\left(\frac{\left[\theta_{y}\right]}{\left[\theta_{x}\right]}\right)
$$

L'application de la transformée de Légendre-Fenchel permet, en établissant la relation reliant les deux fonctions énergies $w^{*}(\boldsymbol{M})$ et $w([\boldsymbol{\theta}])$, l'identification de la fonction rigidité $k(\varphi)$ à partir de la fonction souplesse $s(\Phi)$ issue des calculs tridimensionnels. La loi de comportement de la section fissurée est obtenue par dérivation de la fonction énergie de déformation $w([\boldsymbol{\theta}])$. On écrit alors

$$
\left(\begin{array}{l}
M_{x} \\
M_{y}
\end{array}\right)=\frac{E I}{2 L}\left(\begin{array}{cc}
k(\varphi) & -\frac{1}{2} k^{\prime}(\varphi) \\
\frac{1}{2} k^{\prime}(\varphi) & k(\varphi)
\end{array}\right)\left(\begin{array}{l}
{\left[\theta_{x}\right]} \\
{\left[\theta_{y}\right]}
\end{array}\right), \quad k^{\prime}(\varphi)=\frac{d k(\varphi)}{d \varphi}
$$

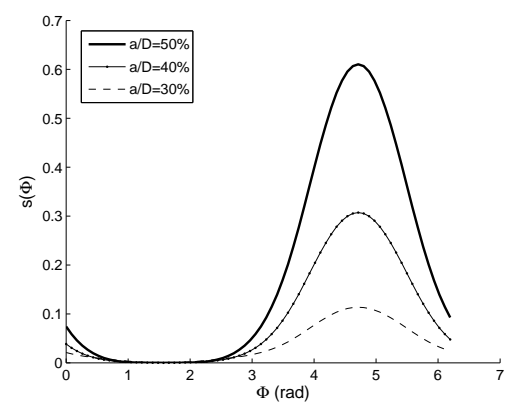

(a) $L=2 D$

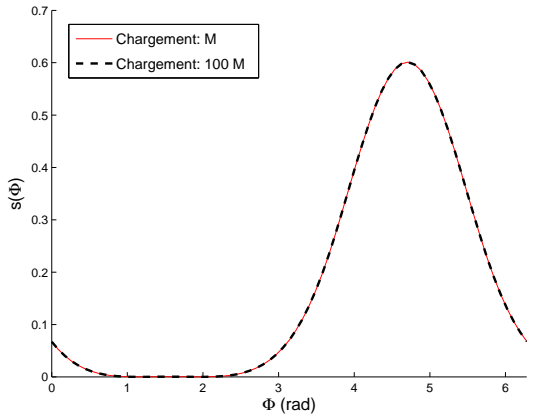

(b) $L=2 D$

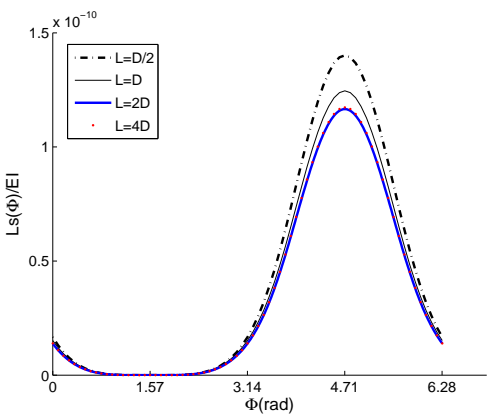

(c)

Figure 2. Souplesses apportées par la section fissurée 


\subsection{Construction d'un élément fini de poutre fissurée}

Il existe deux procédures pour introduire la flexibilité locale due à la fissuration d'une section dans une poutre. La première, consiste en la construction d'une matrice de rigidité exclusivement pour la section fissurée représentée, alors, par un ressort nodal. Il s'agit d'inverser la matrice de souplesse de la section fissurée. Ainsi, lorsque la fissure est très peu profonde, ou lorsque le chargement conduit à sa fermeture totale, la souplesse apportée par la section fissurée est très faible, voire nulle et, par conséquent, les termes de la matrice de rigidité deviennent très élevés conduisant à des problèmes de convergence et des coûts de calculs trop élevés lors de l'intégration numérique du système différentiel résultant (El Arem, 2006; Saavedra et al., 2001).

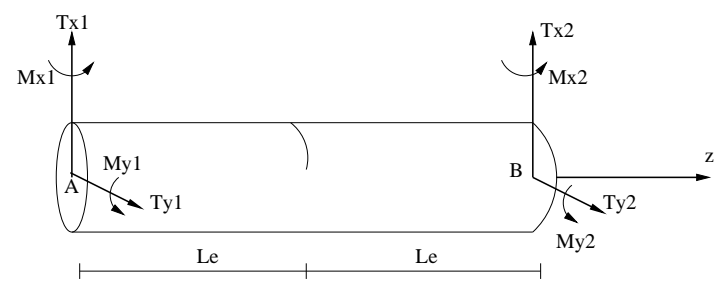

Figure 3. Schéma de l'élément fini de poutre fissurée

La seconde technique, adoptée ici, consiste à construire la matrice de rigidité d'un élément fini de poutre fissurée et de l'assembler avec les éléments représentant les parties saines du système comme dans le cas classique. Ainsi, l'énergie élastique apportée par la section fissurée n'est plus concentrée au niveau de l'élément nodal qui la représente mais répartie sur toute la longueur de l'élément fissuré. Dans des études antérieures (Verrier et al., 2003; El Arem et al., 2003; El Arem, 2006), on a montré que les effets du cisaillement sur le mécanisme de respiration de la fissure sont négligeables et ne seront, par conséquent, pas considérés dans cette étude.

On considère l'élément fini de poutre fissurée de longueur $2 L_{e}$, de section circulaire de diamètre $D$ et de moment d'inertie quadratique $I$, cf. figure 3 . Les déplacements du nœud $A$ sont tous bloqués, on commence alors par établir une relation de la forme

$$
\boldsymbol{u}=\mathcal{S}(\boldsymbol{f}) \cdot \boldsymbol{f}
$$

$\boldsymbol{f}=\left\{T_{x_{2}}, T_{y_{2}}, M_{x_{2}}, M_{y_{2}}\right\}^{t}$ et $\boldsymbol{u}=\left\{u_{x_{2}}, u_{y_{2}}, \theta_{x_{2}}, \theta_{y_{2}}\right\}^{t}$ désignent, respectivement, les vecteurs chargement et déplacement résultants au niveau de la section libre $\left(z=2 L_{e}\right) \cdot \mathcal{S}(\boldsymbol{f})$ est la matrice de souplesse de la structure. 
Au niveau de la section fissurée $\left(z=L_{e}\right)$, les efforts intérieurs sont donnés par:

$$
\left\{\begin{array}{l}
T_{x}=T_{x_{2}} \\
T_{y}=T_{y_{2}} \\
M_{x}=M_{x_{2}}-L_{e} T_{y} \\
M_{y}=M_{y_{2}}+L_{e} T_{x}
\end{array}\right.
$$

La respiration de la fissure est gouvernée par la direction du moment de flexion au niveau de la section fissurée $\boldsymbol{M}=\left(M_{x}, M_{y}\right)$. Soit $\Phi=\operatorname{atan}\left(\frac{M_{y}}{M_{x}}\right)$, l'énergie élastique de l'élément fissuré s'écrit

$$
W^{*}(\boldsymbol{f})=W_{s}^{*}(\boldsymbol{f})+w^{*}(\boldsymbol{M})=W_{s}^{*}(\boldsymbol{f})+\frac{L}{E I}\|\boldsymbol{M}\|^{2} s(\Phi)
$$

$W_{s}^{*}(\boldsymbol{f})$ désigne l'énergie élastique de l'élément fini non fissuré de longueur $2 L_{e}$ et soumis aux mêmes conditions de chargement. En utilisant l'expression [3], l'énergie élastique due à la présence de la fissure est donnée par

$$
w^{*}(\boldsymbol{M})=\frac{L}{E I}\|\boldsymbol{M}\|^{2} s(\Phi)
$$

où $L$ est la demi-longueur de l'élément fissuré tridimensionnel utilisé pour l'identification de la fonction $s(\Phi)$ comme expliqué ci-avant.

La relation de comportement non linéaire reliant les efforts appliqués aux déplacements résultants au niveau de la section libre $\left(z=2 L_{e}\right)$ s'obtient par dérivation de la fonction $W^{*}$ par rapport à $\boldsymbol{f}$, soit, en tenant compte de [8],

$$
\boldsymbol{u}=\mathcal{S}(\boldsymbol{f}) \cdot \boldsymbol{f}=\mathcal{S}(\Phi) \cdot \boldsymbol{f}
$$

avec

$$
\mathcal{S}(\Phi)=\mathcal{S}_{0}+\frac{2 L}{E I}\left(\begin{array}{cccc}
L_{e}^{2} s(\Phi) & -\frac{L_{e}^{2}}{2} s^{\prime}(\Phi) & \frac{L_{e}}{2} s^{\prime}(\Phi) & L_{e} s(\Phi) \\
\frac{L_{e}^{2}}{2} s^{\prime}(\Phi) & L_{e}^{2} s(\Phi) & -L_{e} s(\Phi) & \frac{L_{e}}{2} s^{\prime}(\Phi) \\
-\frac{L_{e}}{2} s^{\prime}(\Phi) & -L_{e} s(\Phi) & s(\Phi) & -\frac{1}{2} s^{\prime}(\Phi) \\
L_{e} s(\Phi) & -\frac{L_{e}}{2} s^{\prime}(\Phi) & \frac{1}{2} s^{\prime}(\Phi) & s(\Phi)
\end{array}\right)
$$

où $\mathcal{S}_{0}$ désigne la matrice de souplesse d'un élément de poutre sain de longueur $2 L_{e}$. En appelant $\left\{u_{B / A}\right\}$, le déplacement relatif de $\mathrm{B}$ par rapport à $\mathrm{A}$, on établit la relation suivante

$$
\left\{T_{x_{2}}, T_{y_{2}}, M_{x_{2}}, M_{y_{2}}\right\}^{t}=(\mathcal{S}(\Phi))^{-1}\left\{u_{B / A}\right\}
$$


652 Revue européenne de mécanique numérique. Volume 16 - n 5/2007

Ensuite, on écrit les relations d'équilibre de l'élément de la figure 3, soit

$$
\left\{\begin{array}{l}
T_{x_{1}}=-T_{x_{2}} \\
T_{y_{1}}=-T_{y_{2}} \\
M_{x_{1}}=-M_{x_{2}}+2 L_{e} T_{y_{2}} \\
M_{y_{1}}=-M_{y_{2}}-2 L_{e} T_{x_{2}}
\end{array}\right.
$$

ou en écriture matricielle

$$
\begin{aligned}
& \left\{T_{x_{1}}, T_{y_{1}}, M_{x_{1}}, M_{y_{1}}, T_{x_{2}}, T_{y_{2}}, M_{x_{2}}, M_{y_{2}}\right\}^{t}=\Pi_{1}\left\{T_{x_{2}}, T_{y_{2}}, M_{x_{2}}, M_{y_{2}}\right\}^{t} \\
& \text { avec } \quad \Pi_{1}=\left(\begin{array}{cccc}
-1 & 0 & 0 & 0 \\
0 & -1 & 0 & 0 \\
0 & 2 L_{e} & -1 & 0 \\
-2 L_{e} & 0 & 0 & -1 \\
1 & 0 & 0 & 0 \\
0 & 1 & 0 & 0 \\
0 & 0 & 1 & 0 \\
0 & 0 & 0 & 1
\end{array}\right)
\end{aligned}
$$

Par ailleurs $\left\{u_{B / A}\right\}$ peut se mettre sous la forme

$$
\begin{gathered}
\left\{u_{B / A}\right\}=\left\{u_{B / A}^{1}, u_{B / A}^{2}, u_{B / A}^{3}, u_{B / A}^{4}\right\} \\
\text { avec } \quad\left\{\begin{array}{l}
u_{x_{2}}=u_{B / A}^{1}+u_{x_{1}}+2 L_{e} \theta_{y_{1}} \\
u_{y_{2}}=u_{B / A}^{2}+u_{y_{1}}-2 L_{e} \theta_{x_{1}} \\
\theta_{x_{2}}=u_{B / A}^{3}+\theta_{x_{1}} \\
\theta_{y_{2}}=u_{B / A}^{4}+\theta_{y_{1}}
\end{array}\right.
\end{gathered}
$$

soit en écriture matricielle

$$
\begin{aligned}
& \left\{u_{B / A}\right\}=\Pi_{2}\left\{u_{x_{1}}, u_{y_{1}}, \theta_{x_{1}}, \theta_{y_{1}}, u_{x_{2}}, u_{y_{2}}, \theta_{x_{2}}, \theta_{y_{2}}\right\}^{t} \\
& \text { où } \quad \Pi_{2}=\left(\begin{array}{cccccccc}
-1 & 0 & 0 & -2 L_{e} & 1 & 0 & 0 & 0 \\
0 & -1 & 2 L_{e} & 0 & 0 & 1 & 0 & 0 \\
0 & 0 & -1 & 0 & 0 & 0 & 1 & 0 \\
0 & 0 & 0 & -1 & 0 & 0 & 0 & 1
\end{array}\right)
\end{aligned}
$$


On remarque que $\Pi_{2}$ n'est autre que la transposée de $\Pi_{1}$ notée, dans ce qui suit, $\Pi_{1}^{t}$.

La matrice de rigidité de l'élément de poutre fissurée, $\boldsymbol{K}_{\boldsymbol{e f}}$, vérifie

$$
\begin{aligned}
& \left\{T_{x_{1}}, T_{y_{1}}, M_{x_{1}}, M_{y_{1}}, T_{x_{2}}, T_{y_{2}}, M_{x_{2}}, M_{y_{2}}\right\}^{t}= \\
& \boldsymbol{K}_{e f}\left\{u_{x_{1}}, u_{y_{1}}, \theta_{x_{1}}, \theta_{y_{1}}, u_{x_{2}}, u_{y_{2}}, \theta_{x_{2}}, \theta_{y_{2}}\right\}^{t}
\end{aligned}
$$

En utilisant [15], on obtient

$$
\boldsymbol{\Pi}_{1}\left\{T_{x_{2}}, T_{y_{2}}, M_{x_{2}}, M_{y_{2}}\right\}^{t}=\boldsymbol{K}_{\boldsymbol{e f}}\left\{u_{x_{1}}, u_{y_{1}}, \theta_{x_{1}}, \theta_{y_{1}}, u_{x_{2}}, u_{y_{2}}, \theta_{x_{2}}, \theta_{y_{2}}\right\}^{t}
$$

ensuite, en tenant compte de [13], on écrit :

$$
\boldsymbol{\Pi}_{\mathbf{1}}(S(\Phi))^{-1}\left\{u_{B / A}\right\}=\boldsymbol{K}_{\boldsymbol{e f}}\left\{u_{x_{1}}, u_{y_{1}}, \theta_{x_{1}}, \theta_{y_{1}}, u_{x_{2}}, u_{y_{2}}, \theta_{x_{2}}, \theta_{y_{2}}\right\}^{t}
$$

Finalement, l'équation [18] conduit à :

$$
\boldsymbol{K}_{e f}=\boldsymbol{\Pi}_{\mathbf{1}}(\mathcal{S}(\Phi))^{-1} \boldsymbol{\Pi}_{1}^{t}
$$

Cependant, dans un code d'éléments finis, il est préférable d'écrire la relation [23] en fonction des inconnues du problème (les déplacements nodaux). Cela nous conduit à établir la relation :

$$
(\mathcal{S}(\Phi))^{-1}=\mathcal{K}\left(\varphi_{e}\right)=\mathcal{K}_{0}-\boldsymbol{K}_{e}\left(\varphi_{e}\right)
$$

permettant de distinguer la matrice de rigidité d'un élément de poutre sain de longueur $2 L_{e}$ et de moment d'inertie $I, \boldsymbol{\Pi}_{1} \mathcal{K}_{\mathbf{0}} \boldsymbol{\Pi}_{\mathbf{1}}^{t}$ de la matrice modélisant les effets de la présence de la section fissurée, $\boldsymbol{\Pi}_{1} \boldsymbol{K}_{\boldsymbol{e}}\left(\varphi_{e}\right) \boldsymbol{\Pi}_{\mathbf{1}}^{t} \cdot \varphi_{e}$ est l'angle défini par $\varphi_{e}=\operatorname{atan}\left(\frac{\theta_{y_{2}}-\theta_{y_{1}}}{\theta_{x_{2}}-\theta_{x_{1}}}\right)$ et $\mathcal{K}_{\mathbf{0}}$ est donnée par (Lalanne et al., 1990)

$$
\mathcal{K}_{\mathbf{0}}=\mathcal{S}_{\mathbf{0}}{ }^{-1}=\frac{E I}{2 L_{e}(1+a)}\left(\begin{array}{cccc}
\frac{3}{L_{e}^{2}} & 0 & 0 & -\frac{3}{L_{e}} \\
0 & \frac{3}{L_{e}^{2}} & \frac{3}{L_{e}} & 0 \\
0 & \frac{3}{L_{e}} & 4+a & 0 \\
-\frac{3}{L_{e}} & 0 & 0 & 4+a
\end{array}\right)
$$

avec $a=\frac{12 E I}{4 \mu k S L_{e}^{2}}$ le coefficient permettant la prise en compte des effets du cisaillement. Pour un élément de poutre classique, on a $a=0$. Par la suite, en exploitant la relation [24], on obtient

$$
\begin{aligned}
\mathbf{K}_{\mathbf{e}}\left(\varphi_{e}\right) & =\mathcal{K}_{\mathbf{0}}-(\mathcal{S}(\Phi))^{-1} \\
& =\frac{E I}{2 L}\left(\begin{array}{cccc}
0 & 0 & 0 & 0 \\
0 & 0 & 0 & 0 \\
0 & 0 & k_{x x}\left(\varphi_{e}\right) & k_{x y}\left(\varphi_{e}\right) \\
0 & 0 & k_{y x}\left(\varphi_{e}\right) & k_{y y}\left(\varphi_{e}\right)
\end{array}\right)
\end{aligned}
$$


avec

$$
\left\{\begin{array}{l}
k_{x x}\left(\varphi_{e}\right)=k_{y y}\left(\varphi_{e}\right)=\frac{L^{2}\left(4 L_{e} s(\Phi)+4 L s^{2}(\Phi)+L s^{\prime 2}(\Phi)\right)}{L_{e}\left(4 L_{e}^{2}+8 L L_{e} s(\Phi)+4 L^{2} s^{2}(\Phi)+L^{2} s^{\prime 2}(\Phi)\right)} \\
k_{x y}\left(\varphi_{e}\right)=-k_{y x}\left(\varphi_{e}\right)=-\frac{2 L^{2} s^{\prime}(\Phi)}{4 L_{e}^{2}+8 L L_{e} s(\Phi)+4 L^{2} s^{2}(\Phi)+L^{2} s^{\prime 2}(\Phi)}
\end{array}\right.
$$

Lorsque $L=L_{e}$, on obtient

$$
\left\{\begin{array}{l}
k_{x x}\left(\varphi_{e}\right)=k_{y y}\left(\varphi_{e}\right)=\frac{4 s(\Phi)+4 s^{2}(\Phi)+s^{\prime 2}(\Phi)}{4+8 s(\Phi)+4 s^{2}(\Phi)+s^{\prime 2}(\Phi)} \\
k_{x y}\left(\varphi_{e}\right)=-k_{y x}\left(\varphi_{e}\right)=-\frac{2 s^{\prime}(\Phi)}{4+8 s(\Phi)+4 s^{2}(\Phi)+s^{\prime 2}(\Phi)}
\end{array}\right.
$$

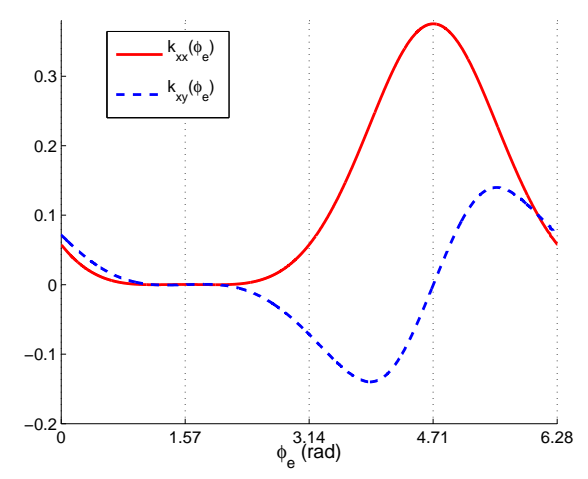

Figure 4. Termes de $\mathbf{K}_{\mathbf{e}}\left(\varphi_{e}\right)$ pour une fissure à fond droit de profondeur $a=\frac{D}{2}$, $L_{e}=L=2 D$

En considérant les résultats des calculs tridimensionnels réalisés sur l'élément fissuré de la figure 1, on identifie la fonction $s(\Phi)$ comme décrit précédemment, ensuite on détermine les termes de la matrice $\mathbf{K}_{\mathbf{e}}\left(\varphi_{e}\right)$ grâce à la relation [26]. Les termes de la matrice $\mathbf{K}_{\mathbf{e}}\left(\varphi_{e}\right)$ pour une fissure à fond droit de profondeur $a=\frac{D}{2}$ sont donnés par la figure 4. Ils montrent de faibles variations sur l'intervalle $\left[0, \frac{\pi}{2}\right]$ tandis que sur $\left[\frac{\pi}{2}, 2 \pi\right]$, leurs variations sont importantes mais régulières. La fissure est totalement fermée pour $\varphi_{e}=\frac{\pi}{2}$, image de $\Phi=\frac{\pi}{2}$. Elle s'ouvre totalement en $\varphi_{e}=\frac{3 \pi}{2}$, image de $\Phi=\frac{3 \pi}{2}$.

\section{Validation de la démarche en statique}

Afin de valider la démarche de construction de l'élément fini de poutre fissurée présentée avant, on se propose de comparer les résultats de la modélisation poutre à 


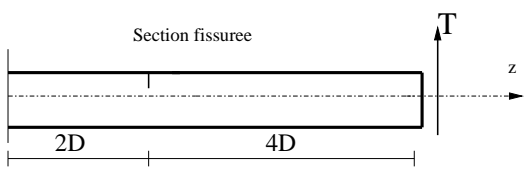

(a) Modèle tridimensionnel

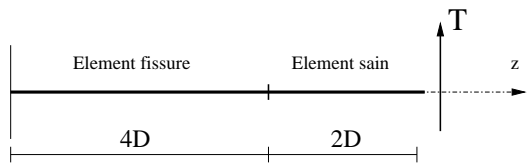

(b) Modèle poutre

Figure 5. Modélisation de la structure par éléments finis $3 D$ et poutre

ceux de la modélisation tridimensionnelle d'une structure comportant une fissure. On considère alors un cylindre d'axe $(o z)$ de diamètre $D=0,5 \mathrm{~m}$, de longueur totale $L_{t}=3 \mathrm{~m}$, encastré à son extrémité $z=0$ et soumis à l'autre au couple d'efforts $\mathbf{T}=\left(T_{x}=\cos (\alpha), T_{y}=\sin (\alpha)\right)$ avec $\alpha$ variant de 0 à $2 \pi$. Le cylindre comporte, en $z=1 \mathrm{~m}$, une fissure à fond droit de profondeur $a=\frac{D}{2}$, cf. figure 5(a). Les calculs tridimensionnels réalisés avec le Code_Aster ${ }^{\circledR}$ tiennent compte du contact unilatéral entre les lèvres de la fissure. Le modèle poutre consiste en deux éléments finis poutre : le premier est un élément de poutre fissurée de longueur totale égale à $2 \mathrm{~m}$, soit $4 D$, cf. figure 5(b). Il comporte une fissure en $z=1 \mathrm{~m}$. Le second est un élément fini de poutre classique de longueur $1 \mathrm{~m}$. La figure 6 montre une très bonne concordance des résultats.

\section{Etude du comportement vibratoire d'un arbre fissuré en flexion}

Considérons, dans ce qui suit, l'arbre fissuré de la figure 7 dont on se propose d'explorer la réponse vibratoire. Il est modélisé par une poutre de masse répartie $m$, de section circulaire constante $S$, de diamètre $D=0,20 \mathrm{~m}$, comportant, à mi-portée, une section fissurée. Il est simplement appuyé à ses deux extrémités et est soumis à l'effet de son poids propre. La rotation imposée à l'arbre se traduit par une vitesse de rotation $\Omega$ de la structure autour de son axe $(O z)$. On se contente de découper cette structure en cinq éléments de même longueur $l=0,80 \mathrm{~m}$. Les éléments $1-2,2-3$, $4-5$ et $5-6$ désignent les parties saines de la structure. L'élément $3-4$ est un élément de poutre fissurée construit selon la démarche détaillée ci-avant.

On suppose que la présence de la fissure n'affecte que la matrice de rigidité du système mécanique dont l'équation de l'équilibre dynamique s'écrit, à tout instant $t$, sous la forme

$$
\mathcal{M X}+\mathcal{D} \dot{\mathrm{X}}+\mathcal{K}(\mathrm{X})=\mathcal{F}
$$

$\mathrm{X}$ désigne le vecteur des déplacements nodaux, $\mathcal{M}$ la matrice de masse, $\mathcal{D}$ la matrice des amortissements visqueux, $\mathcal{K}(\mathrm{X})$ la matrice de rigidité du système et $\mathcal{F}$ le vecteur des forces aux nœuds dues aux seuls effets du poids propre de la structure. $\dot{X}$ est la dérivée par rapport au temps $t$ de la variable $\mathrm{X}$. Dans cette étude aucun balourd résiduel n'a été considéré. Les effets gyroscopiques sont négligeables et, par conséquent, ne seront pas considérés dans cette étude. 

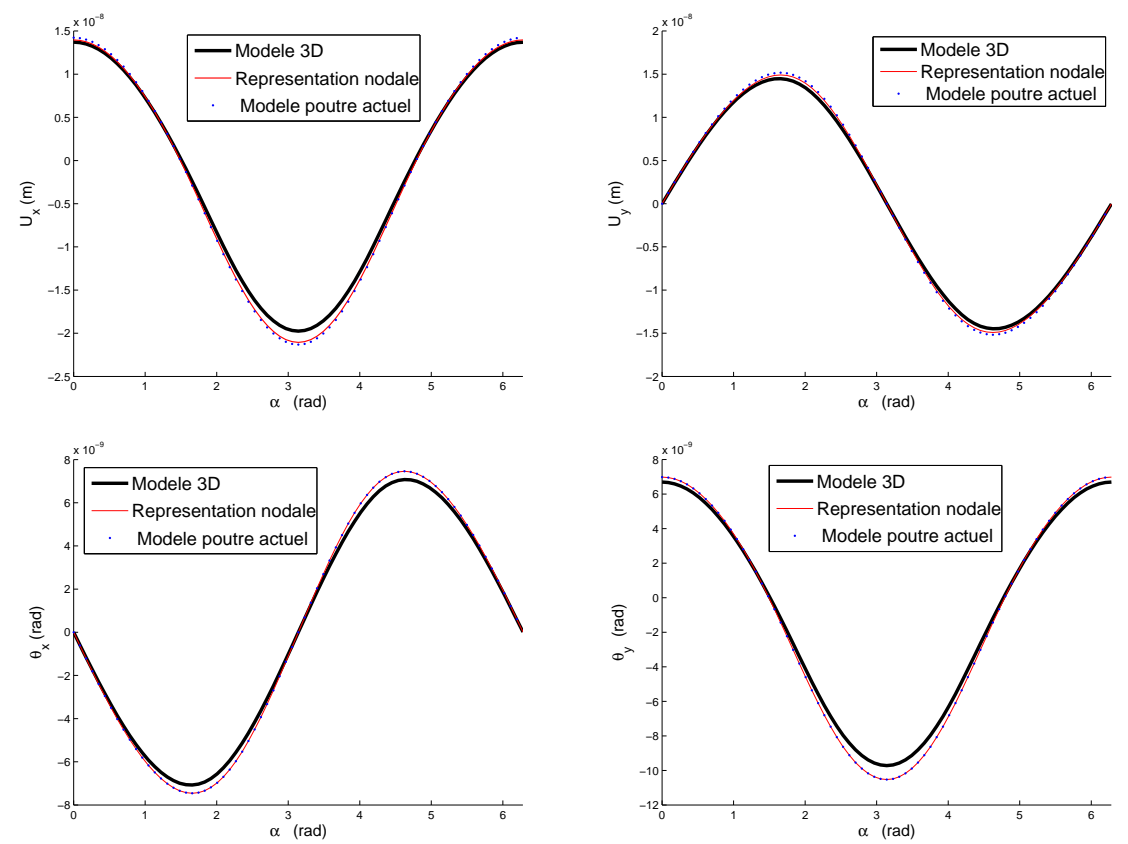

Figure 6. Comparaison du modèle proposé aux résultats tridimensionnels et à ceux de la représentation nodale, $\frac{a}{D}=0,50$

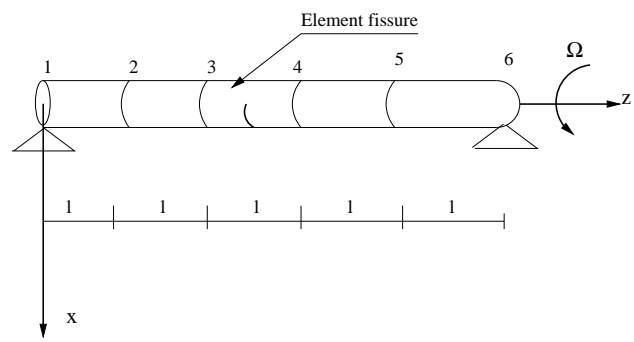

(a) Arbre avec section médiane fissurée

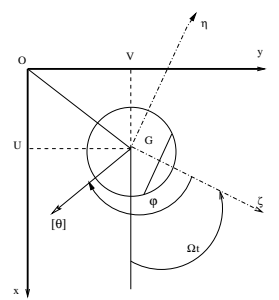

(b) Section fissurée

Figure 7. Modélisation de la structure par éléments finis

A l'instant $t=0$, la fissure est totalement ouverte, cf. figure 7(b). On suppose que la matrice de rigidité reste inchangée entre deux instants $t_{n}=n h$ et $t_{n+1}=(n+1) h$ où $h$ est le pas de temps utilisé pour l'intégration numérique du système dynamique. Afin d'assurer la validité de cette approximation, il faut considérer des pas de temps relativement petits par rapport à la période de la réponse vibratoire du système. 

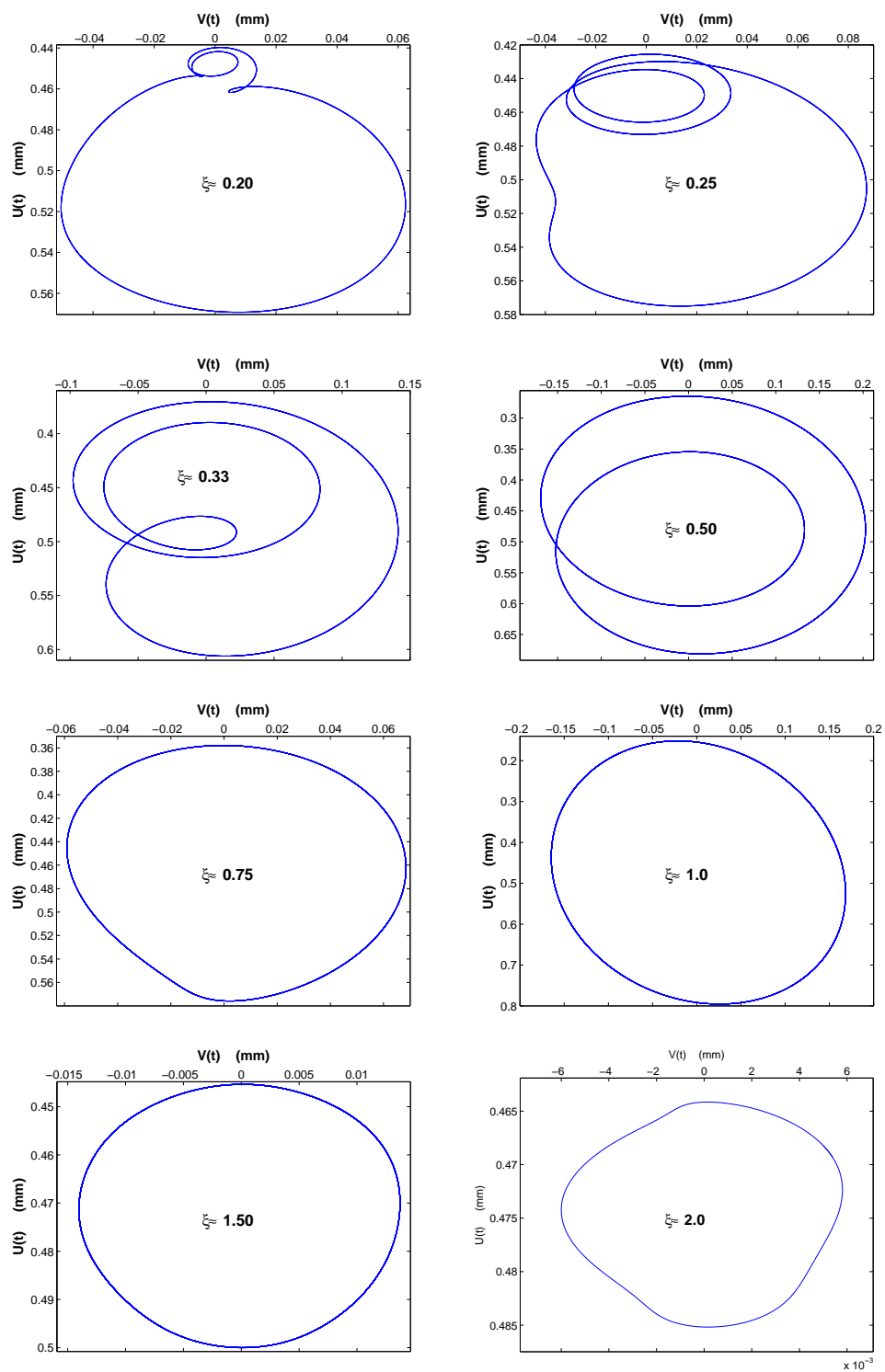

Figure 8. Exemples d'orbites du nœud $3, \frac{a}{D}=0,50, d=0,05$ 
658 Revue européenne de mécanique numérique. Volume 16 - n 5/2007
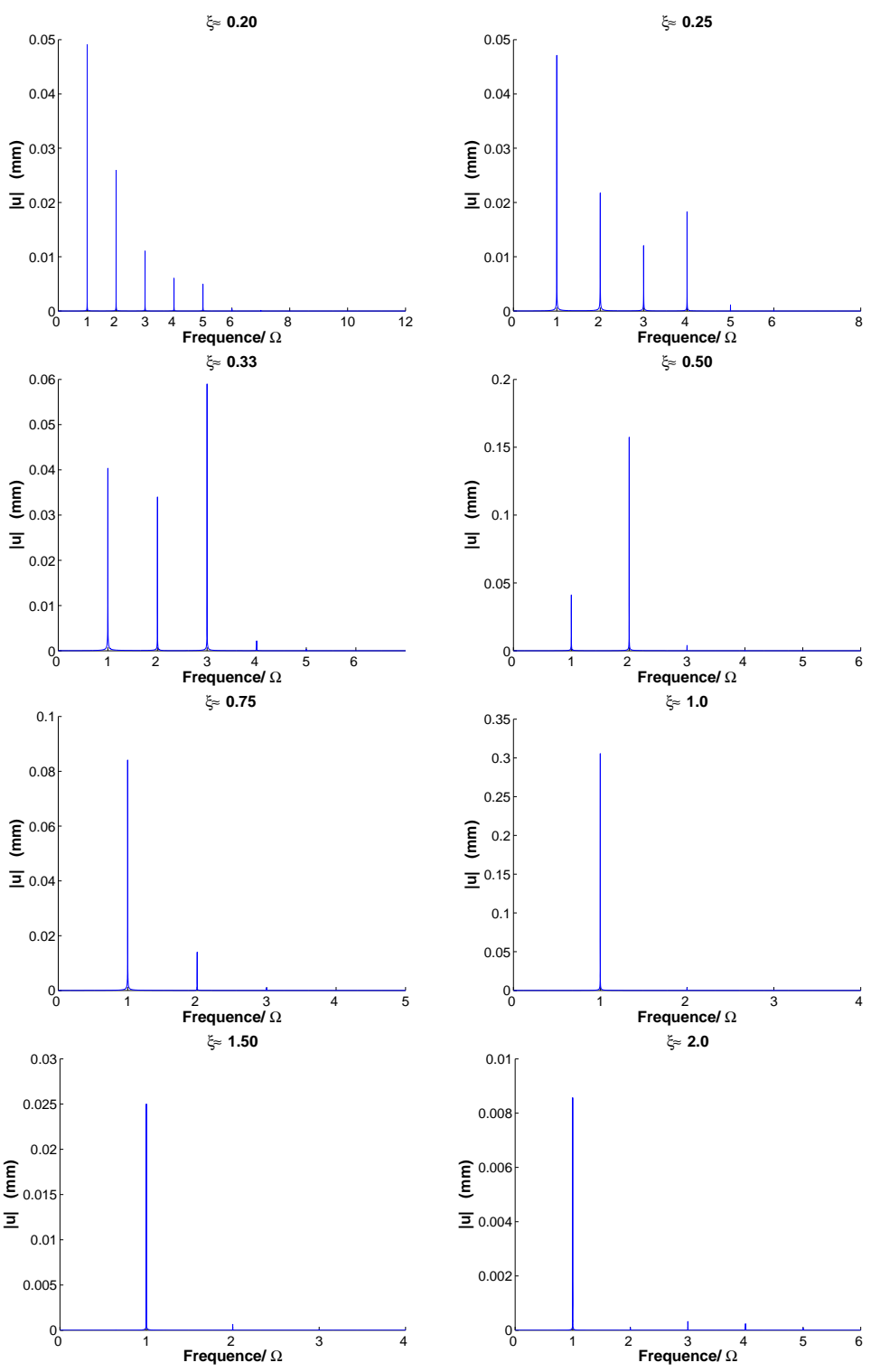

Figure 9. Exemples de spectres d'amplitude de $u(t)$ du nœud $3, \frac{a}{D}=0,50, d=0,05$ 
Dans le domaine des basses fréquences, cette contrainte est facile à surmonter. La matrice de rigidité est alors actualisée à la fin de chaque pas de temps. La méthode d'intégration numérique utilisée ici est celle de HHT (Hilber et al., 1977) avec :

$$
\alpha=\frac{1}{3}, \gamma=\frac{1}{2}+\alpha \text { et } \beta=\frac{1}{4}(1+\alpha)^{2}
$$

ce qui correspond, dans le cas linéaire, à un schéma implicite inconditionnellement stable de précision maximale (Géradin et al., 1992).

La modélisation de la structure fissurée par des éléments finis de type poutre sains connectés par un élément fini de poutre fissurée permet de tenir compte de façon exacte de la condition de contact unilatéral entre les lèvres de la fissure. Ceci se traduit, au moment de la fermeture totale de la fissure, par

$$
\mathbf{K}_{\mathbf{e}}\left(\varphi_{e}\right)=\mathbf{0}
$$

La matrice de rigidité de l'élément fissuré est celle d'un élément sain. De plus, grâce à cette modélisation, les pas de temps utilisés sont 20 à 100 fois plus grands que ceux utilisés pour l'intégration du système dynamique en suivant la méthode implicite-pénalisation utilisée lorsque la section fissurée est modélisée par un élément nodal (longueur nulle) tel que décrit dans (El Arem, 2006) ce qui permet un gain important en temps de calculs et en espace de stockage des résultats numériques.

Le glissement (diminution) des fréquences de résonance de la structure fissurée ne peut à lui seul être considéré comme un indicateur fiable de la présence de fissures (Dimarogonas, 1996; El Arem et al., 2006). L'examen de la réponse vibratoire du système dynamique montre la présence du phénomène de résonance super-harmonique aux passages par des vitesses de rotation de l'arbre $(\Omega)$ voisines de sous-multiples de la première vitesse critique $\left(w_{1}\right)$. Ainsi, pour

$$
\xi \approx \frac{w_{1}}{n} \text { avec } n \in \mathbb{N}
$$

l'orbite ainsi que le diagramme de phase du rotor sont formés de $n$ boucles entrelacées (cf. figures 8 où l'amortissement visqueux réduit $d$ vaut $5 \%$ ). De même, le niveau vibratoire de l'harmonique d'ordre $n$ atteint, à cette vitesse de rotation, des valeurs relativement élevées, cf. figure 9 .

\section{Conclusions}

Une méthode originale de construction d'un élément fini de poutre fissurée a été présentée. Le phénomène de respiration de la fissure est décrit finement puisque la souplesse due à la présence des fissures est déduite de calculs éléments finis tridimensionnels tenant compte des conditions de contact unilatéral entre les lèvres. La démarche est simple et compréhensible et peut être appliquée à toute géométrie de fissures. Il est important de noter la réduction considérable des coûts de calculs par rapport à l'utilisation de la technique de pénalisation. En effet cette technique, employée lorsque la 
section fissurée est modélisée par un élément nodal (El Arem, 2006), conduit à l'apparition de fréquences numériques très élevées (sans sens physique). Les pas de temps considérés pour l'intégration numérique temporelle du système dynamique sont alors très faibles et les coûts de calculs, par conséquent, très importants.

Par rapport à la représentation nodale de la section fissurée et la technique de pénalisation pour tenir compte des conditions de contact entre les lèvres, ce modèle présente les avantages suivants :

- prise en compte de façon exacte du phénomène de respiration de la fissure,

- réduction des coûts de calculs de 20 à 100 fois.

Dans l'étude des arbres fissurés, les chercheurs ont souvent supposé, afin de réduire la difficulté du problème, que l'amplitude des vibrations dues à la présence de fissures est faible par rapport à celle des vibrations dues aux charges permanentes (El Arem et al. (2006), Gasch (1976), Mayes et al. (1976), Mayes et al. (1980), Davies et al. (1984), Henry et al. (1976), Gasch (1993)). Le modèle présenté ici permet de se passer d'une telle hypothèse.

Ce modèle simple et peu coûteux a également permis de mettre en évidence le phénomène de résonance super-harmonique connu pour les systèmes dynamiques tournants présentant des fissures. En revanche, ce phénomène peut avoir d'autres origines telles que la présence de dissymétrie tournante. Ainsi, d'autres paramètres vibratoires doivent être suivis par les exploitants de machines tournantes pour une surveillace efficace de l'état de leurs matériels. Il a été rapporté par El Arem et al. (2006) que les niveaux vibratoires des premières harmoniques ainsi que la déflexion statique moyenne sont deux paramètres sensibles à la propagation de fissures.

\section{Bibliographie}

Abraham O. N. L., Brandon J. A., Cohen A. M., « Remark on the determination of compliance coefficients at the crack section of a uniform beam with circular cross section », J. Sound Vib., vol. 169, n², p. 570-574, 1994.

Andrieux S., Détermination de la loi de comportement en flexion bi-axée d'une poutre fissurée avec prise en compte du contact sur les fissures (in french), rapport interne $\mathrm{n}^{\circ} \mathrm{HP}$ 50/99/018/A, EDF-DER, 2000.

Andrieux S., Varé C., « A 3D cracked beam model with unilateral contact- Application to rotors », European Journal of Mechanics, A/Solids, vol. 21, p. 793-810, 2002.

Anifantis N., Dimarogonas A. D., « Identification of peripheral cracks in cylindrical shells », ASME-Wint. Ann. Meet., Boston, Mass., USA, 1983.

Audebert S., Voinis P., « Comportement dynamique de rotors avec fissuration transverse : modélisation et validation expérimentale (in french) », 13ème colloque Vib. Chocs et bruits, Lyon, France, 2000.

Bachschmid N., Diana N., Pizzigoni B., « The influence of unbalance on cracked rotors », Conf.Vib.Rotat. Mach., U.K, 1980. 
Bachschmid N., Pennacchi P., Tanzi E., Audebert S., « Transverse crack modeling and validationinrotor systems including thermal effects », Int.J. of Rotat. Mach., vol. 10, $\mathrm{n}^{\circ} 4$, p. 253-263, 2004.

Bachschmid N., Pennacchi P., Vania A., « Identificationof multiple faults in rotor systems », Journal of Sound and Vibration, vol. 254(02), p. 327-366, 2002.

Bently D., Muszynska A., « Detection of rotor cracks », 15th Turbomachinery Symposium, Texas, 1986.

Brown F. W., Srawley J. E., « Plane strain crack toughness testing of high strength metallic materials », ASTM STP, vol. 410, p. 12, 1966.

Bueckner H. F., « The propagation of cracks and the energy of elastic deformation », Trans. ASME, vol. 80, p. 1225-1229, 1958.

Darpe A. K., Gupta K., Chawla A., « Transient response and breathing behaviour of a cracked Jeffcott rotor », J. Sound Vib., vol. 272, p. 207-243, 2004.

Davies W., Mayes I., «The vibrational behaviour of multi-shaft, multi-bearing system in the presence of the propagating transverse crack», J. Vib. Acous. Stress Reliab. Design, vol. 106, p. 146-153, 1984.

Dimarogonas A. D., Dynamic response of cracked rotors, Internal report, General Electric Co., Schenectady NY, 1970.

Dimarogonas A. D., Dynamics of cracked shafts, Internal report, General Electric Co., Schenectady NY, 1971.

Dimarogonas A. D., « Crack identification of in aircraft structures », First National aircraft conf., Athens, 1982.

Dimarogonas A. D., « A fuzzy logic neutral network structured expert system shell for diagnosis and prognosis- users manual », EXPERTS, Clayton laboratories, St Louis, Missouri, USA, 1987.

Dimarogonas A. D., « A general purpose rotor dynamic analysis program- users manual », RODYNA, Clayton laboratories, St Louis, Missouri, USA, 1988.

Dimarogonas A. D., « Author's reply », J. Sound and Vibration, vol. 169, p. 575-576, 1994.

Dimarogonas A. D., « Vibration of cracked structures : A state of the art review », Engineering Fracture Mechanics, vol. 55, n 5, p. 831-857, 1996.

Dimarogonas A. D., Paipetis S. A., Analytical methods in rotor dynamics, Applied science Publishers, 1983.

Dirr B. O., Schmalhorst B. K., « Crack depth analysis of a rotating shaft by vibration measurement », ASME J. Vib. Acous. Stres. Relia. Des., vol. 110, p. 158-164, 1988.

El Arem S., Vibrations non-lineaires des structures fissurées : application aux rotors de turbines (in french), $\mathrm{PhD}$ thesis, Ecole Nationale des Ponts et Chaussées, 2006.

El Arem S., Andrieux S., Varé C., Verrier P., « Loi de comportement en flexion d'une section de poutre fissurée avec prise en compte des effets de cisaillement (in french) », 6ème colloque nationale en calcul des structures, vol. II, Giens, p. 223-230, 2003.

El Arem S., Nguyen Q. S., « A simple model for the dynamical behavior of a cracked rotor », Advances in Geomaterials and Structures, Hammamet, p. 393-398, 2006.

Entwistle R. D., Stone B. J., « Survey of the use of vibration methods in the assessment of component geometry », Vib. and Noise Meas. Pred. and Cont., Inst. engineers, Australia, vol. 90, p. 210-217, 1990 . 
662 Revue européenne de mécanique numérique. Volume 16 - n 5/2007

Friswell M., Penny J., « Crack Modelling for Structural Health Monitoring », International Journal of Structural Health Monitoring, vol. 1, p. 139-148, 2002.

Gasch R., « Dynamical behavior of a simple rotor with a cross-sectional crack », Vibrations in rotating machinery, I. Mech. E. Conference, London, p. 123-128, 1976.

Gasch R., " A survey of the dynamic behavior of a simple rotating shaft with a transverse crack», J. Sound and vibration, vol. 160, p. 313-332, 1993.

Géradin M., Rixen D., Théorie des vibrations : Application à la dynamique des structures, Masson, Paris, 1992.

Go C. G., Lin Y. S., « Infinitely small element for dynamic problem of cracked beam », Eng. Fract. Mech., vol. 48, n 4, p. 475-482, 1994.

Gross B., Srawley J. E., Stress intensity factors for a single-edge-notch tension specimen by boundary collocation of a stress function, Tech. Note $n^{\circ}$ D-2395, NASA, 1964.

Gross B., Srawley J. E., Stress intensity factors for a single-edge-notch specimen in bending or combined bending and tension by boundary collocation of a stress function, Tech. Note $\mathrm{n}^{\circ}$ D-2603, NASA, 1965.

Henry T. A., Okah-Avae, « Vibrations in cracked shafts », Vibrations in rotating machinery, Inst. Mech. E. Conference, London, p. 15-19, 1976.

Hilber H. M., Hughes T., Taylor R., « Improved numerical dissipation for time integration algorithms in structural dynamics », Earthquake Engneering and Structural Dynamics, vol. 5, p. 283-293, 1977.

Imam I., Azzaro S. H., Bankert R. J., Scheibel I., « Developement of an on-line rotor crack detection and monitoring system », J. Sound and Vib., vol. 111, p. 241-250, 1989.

Irwin G. R., « Analysis of stresses and strains near the end of a cracktraversing a plate », $J$. App. Mech., vol. 24, p. 361-364, 1957.

Jun O. S., Eun H. J., Earmme Y., Lee C., « Modelling and vibration analysis of a simple rotor with a breathing crack », J. Sound and Vibration, vol. 155, n² 2, p. 273-290, 1992.

Lalanne M., Ferraris G., Rotordynamics Prediction in Engineering, John Wiley \& Sons, England, 1990.

Liebowitz H., Claus D. W., « Carrying capacity of notched columns », Eng. Fract. Mech., vol. 1, p. 379-384, 1968.

Liebowitz H., Vanderveldt H., Harris W. D., « Failure of notched columns », Int. J. Solids Struct., vol. 3, p. 489-500, 1967.

Mayes I., Davies W., « The vibrational behaviour of a rotating shaft system containing a transverse crack », Vibrations in rotating machinery, Inst. Mech. E. Conference, London, p. 5365, 1976.

Mayes I., Davies W., « A method of calculating the vibrational behaviour of coupled rotating shafts containning a transverse crack », Vibrations in rotating machinery, Inst. Mech. E. Conference, London, p. 17-27, 1980.

Okamura H., Liu H. W., Chu C.-S., Liebowitz H., « A cracked column under compression », Eng. Fract. Mech., vol. 1, p. 547-564, 1969.

Ostachowicz W. M., Krawwczuk M., « Vibration analysis of a cracked beam », Comput. Struct., vol. 36, n², p. 245-250, 1990.

Ostachowicz W. M., Krawwczuk M., « Coupled torsional and bending vibrations of a rotor with an open crack », Arch. App. Mech., vol. 62, p. 191-201, 1992. 
Papadopoulos C. A., Dimarogonas A. D., « Coupled longitudinal and bending vibrations of a rotating shaft with an open crack», J. Sound vibration, vol. 117, p. 81-93, 1987a.

Papadopoulos C. A., Dimarogonas A. D., « Coupling of bending and torsional vibration of a cracked Timoshenko beam », Ing. Arch., vol. 57, p. 496-505, $1987 \mathrm{~b}$.

Papadopoulos C. A., Dimarogonas A. D., « Stability of cracked rotors in the coupled vibration mode », ASME-11th Bien. Conf. Mech. Vib. Noise, Boston, Mass., USA, p. 25-34, 1987c.

Rice J. R., Levy N., « The part-through surface crack in an elastic plate», J. App. Mech., vol. 39, p. 185-194, 1972.

Saavedra P. N., Cuitino L. A., « Crack detection and vibrational behavior of cracked beams », Comput. Struct., vol. 79, p. 1451-1459, 2001.

Sinou J. J., Lees A. W., « The influence of cracks in rotating shafts », J. Sound Vib., vol. 285, p. 1015-1037, 2005.

Sinou J. J., Lees A. W., « A non-linear study of a cracked rotor », European Journal of Mechanics-A/Solids, vol. 26, p. 152-170, 2007.

Tada H., Paris P., Irwin G., The stress analysis of cracks handbook, Del Research Corporation, Hellertown, pennsylvania, USA, 1973.

Varé C., Andrieux S., « Modélisation d'une section de poutre fissurée-Application aux rotors de turbine », Revue Française de Mécanique, vol. 2000, n² 2, p. 91-97, 2000.

Verrier P., El Arem S., Calcul d'un modèle de rotor fissuré avec prise en compte de l'effort tranchant-cas de la flexion plane (in french), rapport interne $n^{\circ}$ HT-65/03/009/A, EDF-DER, 2003.

Wauer J., « On the dynamics of cracked rotors : A literature survey », Applied Mechanical Reviews, vol. 43, n 1, p. 13-17, 1990.

Westmann R. A., Yang W. H., « Stress analysis of cracked rectangular beams », J. APP. Mech., vol. 32, p. 639-701, 1967.

Zuo L., Etude du comportement dynamique des systèmes linéaires par morceaux- contribution à la détection des fissures dans les arbres de machines tournantes, PhD thesis, EPFL, 1992.

Zuo L., Curnier A., « Nonlinear real and complex modes of conewise linear systems », J. Sound and Vib., vol. 174, n 3, p. 289-313, 1994. 
